\title{
EMD: A Package for Empirical Mode Decomposition and Hilbert Spectrum
}

\author{
by Donghoh Kim and Hee-Seok Oh
}

\section{Introduction}

The concept of empirical mode decomposition (EMD) and the Hilbert spectrum (HS) has been developed rapidly in many disciplines of science and engineering since Huang et al. (1998) invented EMD. The key feature of EMD is to decompose a signal into so-called intrinsic mode function (IMF). Furthermore, the Hilbert spectral analysis of intrinsic mode functions provides frequency information evolving with time and quantifies the amount of variation due to oscillation at different time scales and time locations. In this article, we introduce an $\mathrm{R}$ package called EMD (Kim and Oh, 2008) that performs oneand two- dimensional EMD and HS.

\section{Intrinsic mode function}

The essential step extracting an IMF is to identify an oscillation embedded in a signal from local time scale. Consider the following synthetic signal $x(t), 0<t<9$ of the form

$$
x(t)=0.5 t+\sin (\pi t)+\sin (2 \pi t)+\sin (6 \pi t) .
$$

The signal in Figure 1 consists of several components, which are generated through the process that a component is superimposed to each other.

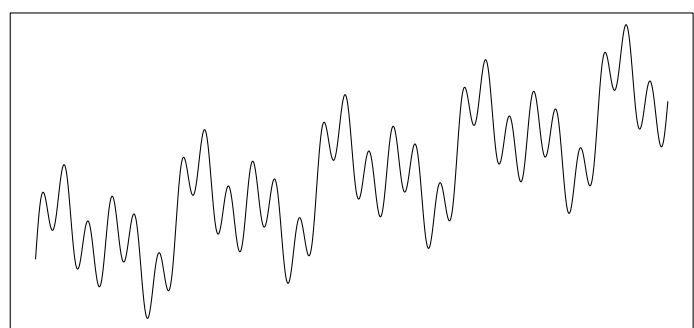

Figure 1: A sinusoidal function having 4 components

An intrinsic oscillation or frequency of a component, for example, $\sin (\pi t), t \in(0,9)$ in Figure 1 can be perceived through the red solid wave or the blue dotted wave in Figure 2. The blue dotted wave in Figure 2 illustrates one cycle of intrinsic oscillation which starts at a local maximum and terminates at a consecutive local maximum by passing through two zeros and a local minimum which eventually appears between two consecutive maxima. A component for a given time scale can be regarded as the composition of repeated intrinsic oscillation which is symmetric to its local mean, zero.

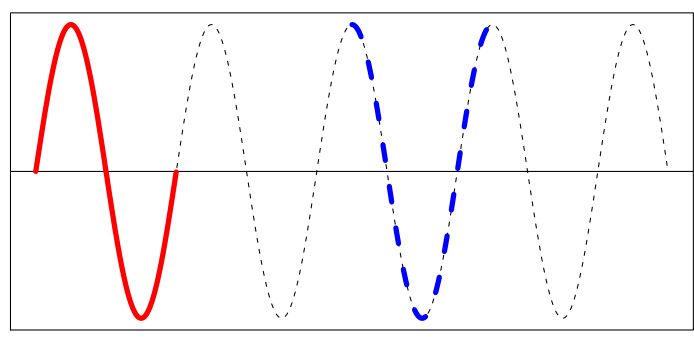

Figure 2: A sinusoidal function

Thus the first step to define intrinsic oscillation is to detect local extrema or zero-crossings. The function extrema () identifies local extrema and zerocrossings of the signal in Figure 2.

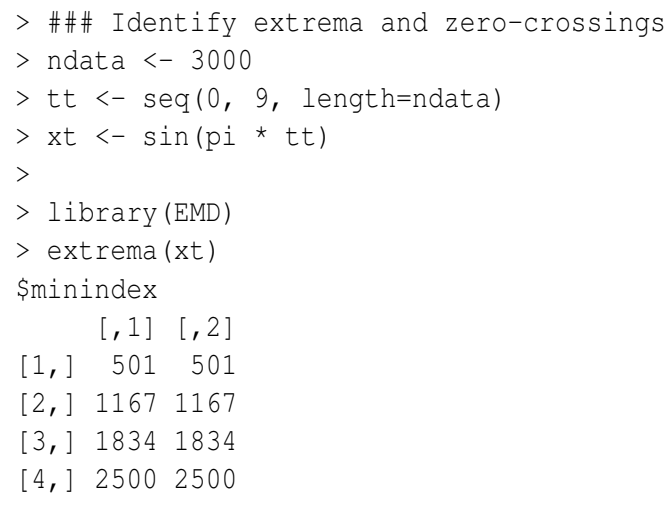

\$maxindex

$[, 1][, 2]$

$[1] \quad 168 \quad$,

$[2] \quad 834 \quad$,

$[3]$,

$[4]$,

$[5]$,

\$nextreme

[1] 9

\$cross

$[, 1][, 2]$

$[1] \quad 1 \quad$,

$[2] \quad 334 \quad$,

$[3] \quad 667 \quad$,

[4, ] 10001001

$[5]$,

[6, ] 16671668

[7, ] 20002001

$[8]$,

$[9]$,

\$ncross

[1] 9

The function extrema () returns a list of followings. 
- minindex : matrix of time index at which local minima are attained. Each row specifies a starting and ending time index of a local minimum.

- maxindex : matrix of time index at which local maxima are attained. Each row specifies a starting and ending time index of a local maximum.

- nextreme : the number of extrema.

- cross : matrix of time index of zero-crossings. Each row specifies a starting and ending time index of zero-crossings.

- ncross: the number of zero-crossings.

Once local extrema is obtained, the intrinsic mode function is derived through the sifting procedure.

\section{Sifting process}

Huang et al. (1998) suggested a data-adapted algorithm extracting a sinusoidal wave or equivalently a frequency from a given signal $x$. First, identify the local extrema in Figure 3(a), and generate the two functions called the upper envelope and lower envelope by interpolating local maxima and local minima, respectively. See Figure 3(b). Second, take their average, which will produce a lower frequency component than the original signal as in Figure 3(c). Third, by subtracting the envelope mean from the signal $x$, the highly oscillated pattern $h$ is separated as in Figure 3(d).

Huang et al. (1998) defined an oscillating wave as an intrinsic mode function if it satisfies two conditions 1) the number of extrema and the number of zero-crossings differs only by one and 2) the local average is zero. If the conditions of IMF are not satisfied after one iteration of aforementioned procedure, the same procedure is applied to the residue signal as in Figure 3(d), (e) and (f) until properties of IMF are satisfied.

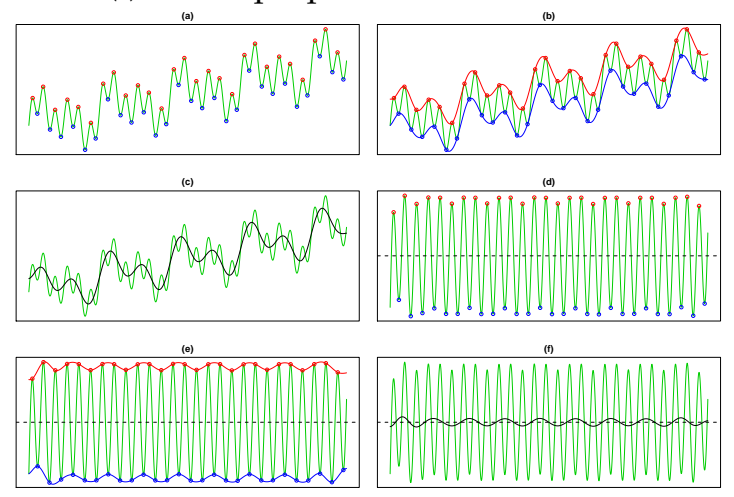

Figure 3: Sifting procedure

This iterative process is called sifting. The following code produces Figure 3, and the function extractimf() implements the sifting algorithm by identifying the local extrema with the extrema(). Note that when setting the option 'check=TRUE' , one must click the plot to proceed to the next step.

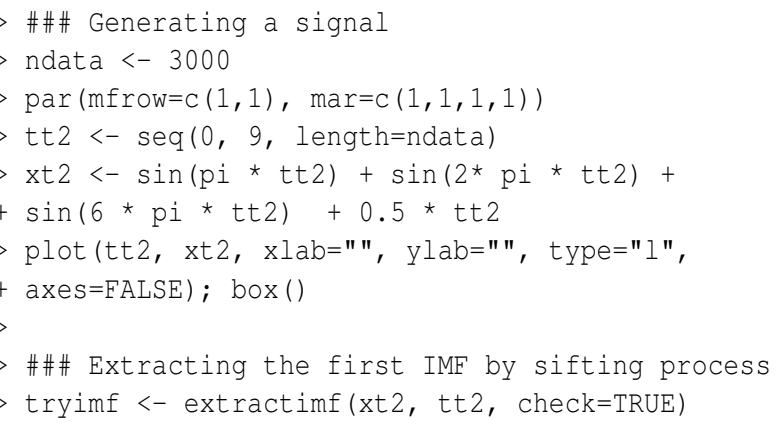

The function extractimf() extracts IMF's from a given signal, and it is controlled by the following arguments.

- residue : observation or signal observed at time tt.

- tt : observation index or time index.

- tol : tolerance for stopping rule.

- max.sift : the maximum number of sifting.

- stoprule : stopping rule.

- boundary : specifies boundary condition.

- check : specifies whether the sifting process is displayed. If check=TRUE, click the plotting area to start the next step.

\section{Stopping rule}

The sifting process stops when the replication of sifting procedure exceed the predefined maximum number by max.sift or satisfies the properties of IMF by stopping rule. The stopping rule stoprule has two options - "type1" and "type2". The option stoprule = "type1" makes the sifting process stop when the absolute values of the candidate IMF $h_{i}$ are smaller than tolerance level, that is, $\left|h_{i}(t)\right|<$ tol for all $t$. Or by the option stoprule $=$ "type2", the sifting process stops when the variation of consecutive candidate IMF's is within the tolerance level,

$$
\sum_{t}\left(\frac{h_{i}(t)-h_{i-1}(t)}{h_{i-1}(t)}\right)^{2}<\text { tol. }
$$

\section{Boundary adjustment}

To eliminate the boundary effect of a signal, it is necessary to adjust a signal at the boundary. Huang et al. (1998) extended the original signal by adding artificial waves repeatedly on both sides of the boundaries. The waves called characteristic waves are constructed by repeating the implicit mode formed from extreme values nearest to boundary. The argument boundary specifies the adjusting method of the 
boundary. The argument boundary = "wave" constructs a wave which is defined by two consecutive extrema at either boundary, and adds four waves at either end. Typical adjusting method extends a signal assuming that a signal is symmetric or periodic. The option boundary = "symmetric" or boundary = "periodic" extends both boundaries symmetrically or periodically.

Zeng and He (2004) considered two extended signals by adding a signal in a symmetric way and reflexive way called even extension and odd extension, respectively. Even extension and odd extension produce the extended signals so that its average is zero. This boundary condition can be specified by boundary = "evenodd". For each extended signal, upper and lower envelopes are constructed and envelope mean of the extended signals is defined by the average of four envelopes. Then, the envelope mean outside the time scale of the original signal is close to zero, while the envelope mean within the time scale of the original signal is almost the same as the envelope mean of the original signal. On the other hand, the option boundary = "none" performs no boundary adjustments.

\section{Empirical mode decomposition}

Once the highest frequency is removed from a signal, the same procedure is applied on the residue signal to identify next highest frequency. The residue is considered a new signal to decompose.

Suppose that we have a signal from model (1). The signal in Figure 1 is composed of 4 components from $\sin (6 \pi t)$ with the highest frequency to $0.5 t$ with the lowest frequency. We may regard the linear component as a component having the lowest frequency. The left panel in Figure 4 illustrates the first IMF and the residue signal obtained by the function extractimf(). If the remaining signal is still compound of components with several frequencies as in the left panel in Figure 4, then the next IMF is obtained by taking the residue signal as a new signal in the right panel in Figure 4. The number of extrema will decrease as the procedure continues, so that the signal is sequently decomposed into the highest frequency component $i m f_{1}$ to the lowest frequency component $i m f_{n}$, for some finite $n$ and a residue signal $r$. Finally, we have $n$ IMF's and a residue signal as

$$
x(t)=\sum_{i=1}^{n} i m f_{i}(t)+r(t) .
$$

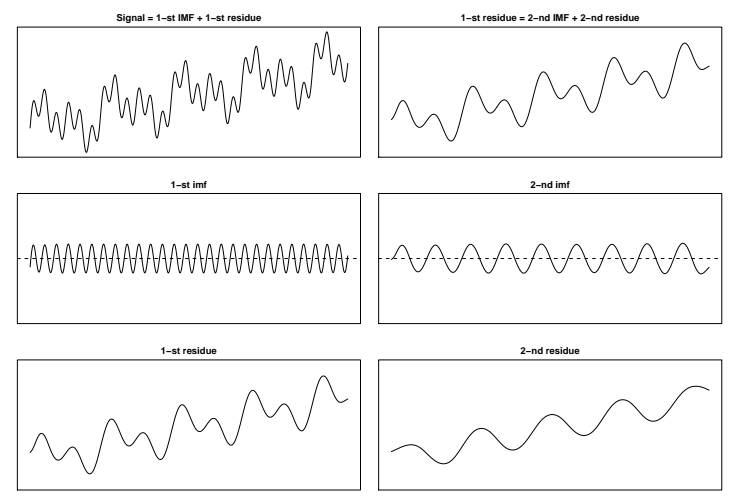

Figure 4: Two IMF's by the sifting algorithm

The above-mentioned decomposition process is implemented by the function emd () that utilizes the functions extractimf() and extrema(). The final decomposition result by the following code is illustrated in Figure 5.
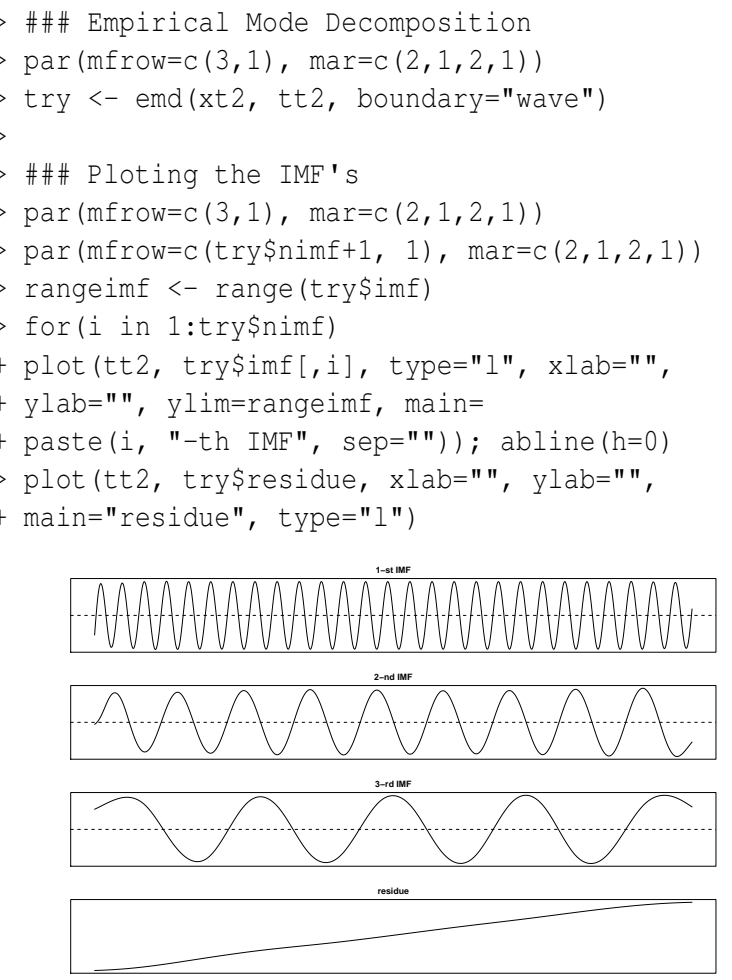

Figure 5: Decomposition of a signal by model (1)

The arguments of emd () are similar to those of extractimf (). The additional arguments are

- max.imf : the maximum number of IMF's.

- plot.imf : specifies whether each IMF is displayed. If plot.imf=TRUE, click the plotting area to start the next step.

Up to now we have focused on artificial signals without any measurement error. A typical signal in the real world is corrupted by noise, which is not the component of interest and contains no interpretable 
information. A remedy to smooth out the noise is to apply smoothing technique not interpolation during the sifting process. Then the first IMF might capture the entire noise effectively. As an alternative, Kim and $\mathrm{Oh}$ (Kim and Oh, 2006) proposed an efficient smoothing method for IMF's by combining the conventional cross-validation and thresholding approach. By thresholding, noisy signal can be denoised while the distinct localized feature of a signal can be kept.

\section{Intermittence}

Huang et al. $(1998,2003)$ pointed out that intermittence raises mode mixing, which means that different modes of oscillations coexist in a single IMF. Since EMD traces the highest frequency embedded in a given signal locally, when intermittence occurs, the shape of resulting IMF is abruptly changed and this effect distorts procedures thereafter.

Huang et al. (2003) attacked this phenomenon by restricting the size of frequency. To be specific, the distance limit of the successive maxima (minima) in an IMF is introduced. Thus, IMF composes of only sinusoidal waves whose length of successive maxima (minima) are shorter than their limit. Equivalently, we may employ the length of the zero-crossings to overcome the intermittence problem. Consider a signal $x(t)$ combined by two sine curves (Deering and Kaiser, 2005),

$$
\begin{aligned}
& x(t) \\
& = \begin{cases}\sin \left(2 \pi f_{1} t\right)+\sin \left(2 \pi f_{2} t\right), & \frac{1}{30} \leq t \leq \frac{2}{30} \\
\sin \left(2 \pi f_{1} t\right), & \text { otherwise }\end{cases}
\end{aligned}
$$

Figure 6 illustrates the signal $x(t)$ when $f_{1}=1776$ and $f_{2}=1000$ and the corresponding two IMF's. The first IMF absorbs the component that appeared in the second IMF between $\frac{1}{30}$ and $\frac{2}{30}$. Thus, the resulting IMF has a mode mixing pattern.

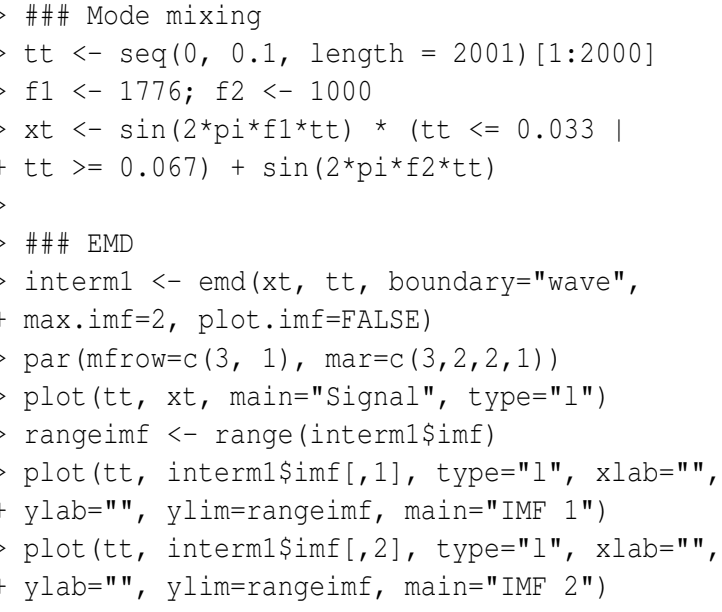

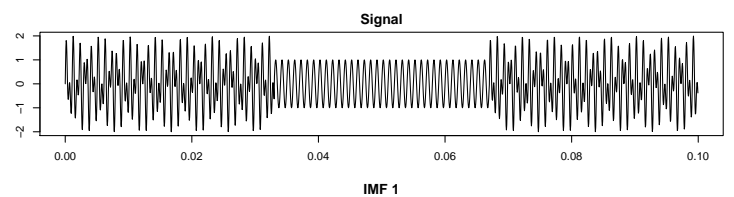
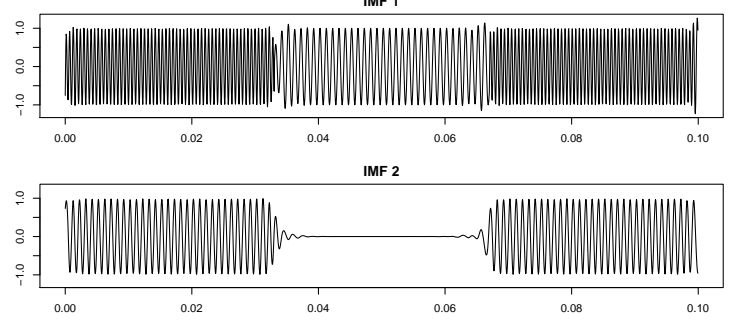

Figure 6: Signal $x(t)$ by model (2) and first two IMF's

By following the approach of Huang et al. (1998, 2003), we can remove waves whose empirical period represented by the distance of other zero-crossings is larger than 0.0007 in the first IMF. The period information obtained by histogram in Figure 7 can be used to choose an appropriate distance. We eliminate the waves with lower frequency in the first IMF with the histogram of other zero-crossings.
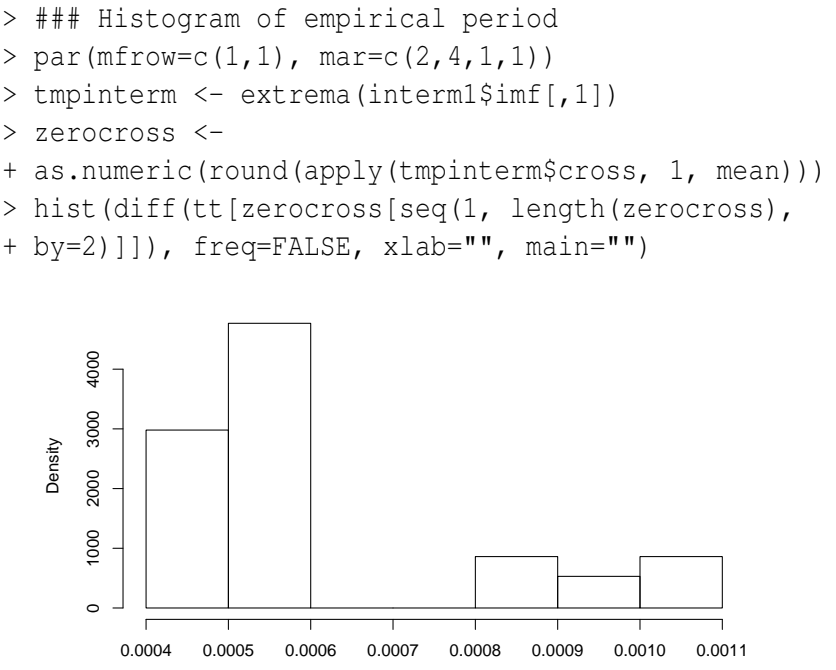

Figure 7: Histogram of the empirical period

Figure 8 shows the resulting IMF's after treating intermittence properly. The argument interm of the function emd () specifies a vector of periods to be excluded from the IMF's.

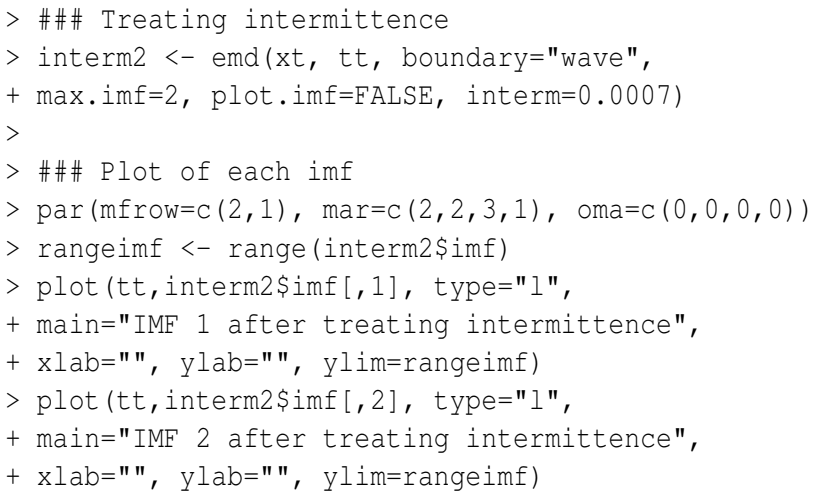




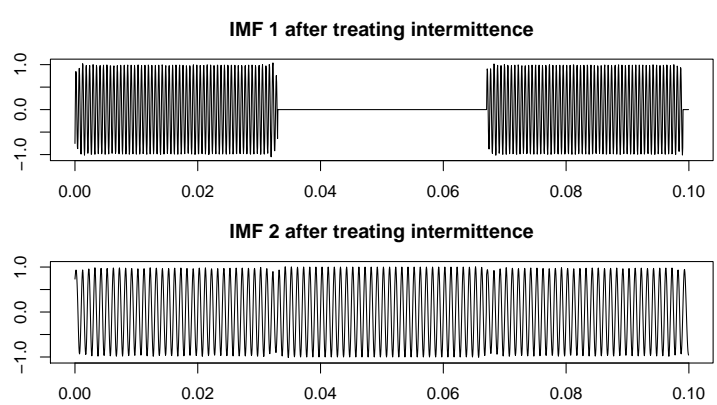

Figure 8: Decomposition of signal $x(t)$ by model (2) after treating the intermittence.

\section{Hilbert spectrum}

When a signal is subject to non-stationarity so that the frequency and amplitude change over time, it is necessary to have a more flexible and extended notion of frequency. Huang et al. (1998) used the concept of instantaneous frequency through the Hilbert transform. For a comprehensive explanation of the Hilbert transform, refer to Cohen (1995). For a real signal $x(t)$, the analytic signal $z(t)$ is defined as $z(t)=x(t)+i y(t)$ where $y(t)$ is the Hilbert transform of $x(t)$, that is, $y(t)=\frac{1}{\pi} P \int_{-\infty}^{\infty} \frac{x(s)}{t-s} d s$ where $P$ is the Cauchy principal value. The polar coordinate form of the analytic signal $z$ with amplitude and phase is $z(t)=a(t) \exp (i \theta(t))$ where amplitude $a(t)$ is $\|z(t)\|=\sqrt{x(t)^{2}+y(t)^{2}}$ and phase $\theta(t)$ is $\arctan \left(\frac{y(t)}{x(t)}\right)$. The instantaneous frequency as timevarying phase is defined as $\frac{d \theta(t)}{d t}$. After decomposing a signal into IMF's with EMD thereby preserving any local property in the time domain, we can extract localized information in the frequency domain with the Hilbert transform and identify hidden local structures embedded in the original signal. The local information can be described by the Hilbert spectrum which is amplitude and instantaneous frequency representation with respect to time. Figure 9 describes the Hilbert spectrum for IMF 1 of the signal of model (2) before and after treating the intermittence. The $X-Y$ axis represents time and instantaneous frequency, and the color intensity of the image depicts instantaneous amplitude.

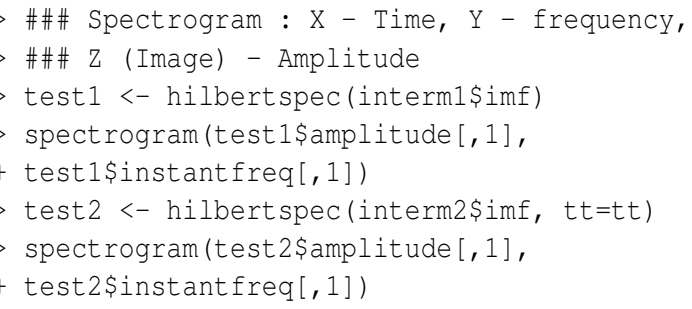

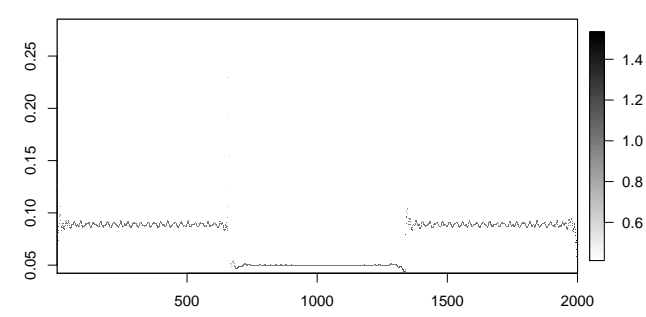

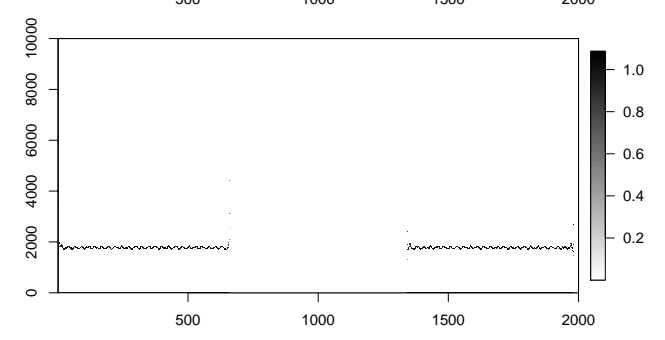

Figure 9: The Hilbert spectrum for IMF 1 of the signal of model (2)

For multiple signals, the function hilbertspec () calculates the amplitudes and instantaneous frequency using Hilbert transform. The function has the following arguments,

- xt : matrix of multiple signals. Each column represents a signal.

- tt : observation index or time index.

The function hilbertspec () returns a matrix of amplitudes and instantaneous frequencies for multiple signals. The function spectrogram() produces an image of amplitude by time index and instantaneous frequency. The horizontal axis represents time, the vertical axis is instantaneous frequency, and the color of each point in the image represents amplitude of a particular frequency at a particular time. It has arguments as

- amplitude : vector or matrix of amplitudes for multiple signals.

- freq : vector or matrix of instantaneous frequencies for multiple signals.

- tt : observation index or time index.

- multi : specifies whether spectrograms of multiple signals are separated or not.

- nlevel : the number of color levels used in legend strip

- size : vector of image size.

\section{Extension to two dimensional im- age}

The extension of EMD to an image or two dimensional data is straightforward except the identification of the local extrema. Once the local extrema 
are identified, the two dimensional smoothing spline technique is used for the sifting procedure.

For the two-dimensional case, we provide four $\mathrm{R}$ functions.

(1) extrema $2 \mathrm{dC}$ () for identifying the two dimensional extrema,

(2) extractimf $2 d$ () for extracting the IMF from a given image,

(3) emd2d () for decomposing an image to $I_{M F}$ s and the residue image combining two $R$ functions above, and

(4) imageEMD ( ) for displaying the decomposition results.

As in a one-dimensional case, extractimf2d() extracts two dimensional IMF's from a given image based on local extrema identified by extrema2dC (). Combining these functions, emd2d() performs decomposition and its arguments are as follows.

- $\mathrm{z}$ : matrix of an image observed at $(\mathrm{x}, \mathrm{y})$.

- $x, y$ : locations of regular grid at which the values in $z$ are measured.

- tol : tolerance for stopping rule of sifting.

- max.sift : the maximum number of sifting.

- boundary : specifies boundary condition 'symmetric', 'reflexive' or 'none'.

- boundperc : expand an image by adding specified percentage of image at the boundary when boundary condition is 'symmetric' or 'reflexive'.

- $\max . i m f:$ the maximum number of IMF.

- plot.imf : specifies whether each IMF is displayed. If plot.imf=TRUE, click the plotting area to start the next step.

The following $\mathrm{R}$ code performs two dimensional EMD of the Lena image. The size of the original image is reduced for computational simplicity.

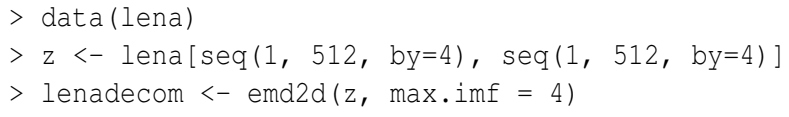

The $\mathrm{R}$ function imageEMD () plots decomposition results and the argument extrma=TRUE illustrates the local maxima (minima) with the white (black) color and grey background. See Figure 10.

\footnotetext{
$>\operatorname{imageEMD}(z=z, e m d z=l$ enadecom, extrema=TRUE,
}

$+\operatorname{col}=\operatorname{gray}(0: 100 / 100))$

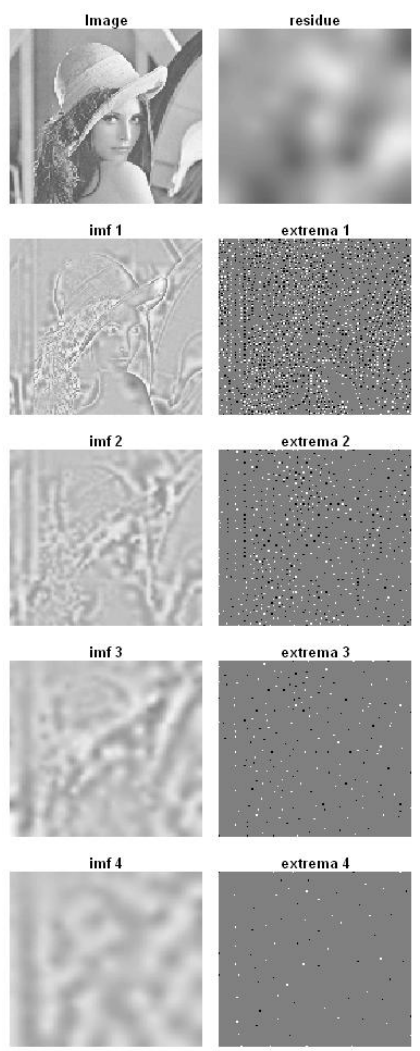

Figure 10: Decomposition of the Lena image

\section{Conclusions}

IMF's through EMD provide a multi-resolution tool and spectral analysis gives local information with time-varying amplitude and phase according to the scales. We introduce EMD, an $\mathrm{R}$ package for the proper implementation of EMD, and the Hilbert spectral analysis for non-stationary signals. It is expected that R package EMD makes EMD methodology practical for many statistical applications.

\section{Bibliography}

L. Cohen. Time-Frequency Analysis. Prentice-Hall, 1995.

R. Deering and J. F. Kaiser. The Use of a Masking Signal to Improve Empirical Mode Decomposition. Proceedings of IEEE International Conference on Acoustics, Speech, and Signal Processing, 4:485-488, 2005.

N. E. Huang, Z. Shen, S. R. Long, M. C. Wu, H. H. Shih, Q. Zheng, N.-C. Yen, C. C. Tung, and H. H. Liu. The Empirical Mode Decomposition and Hilbert Spectrum for Nonlinear and Nonstationary Time Series Analysis. Proceedings of the Royal Society London A., 454:903-995, 1998.

N. E. Huang, M. C. Wu, S. R. Long, S. Shen, W. Qu, P. Gloerson, and K. L. Fan. A Confidence Limit for 
the Empirical Mode Decomposition and Hilbert Spectral Analysis. Proceedings of the Royal Society London A., 31:417-457, 2003.

D. Kim and H-S. Oh. Hierarchical Smoothing Technique by Empirical Mode Decomposition. The Korean Journal of Applied Statistics, 19:319-330, 2006.

D. Kim and H-S. Oh. EMD: Empirical Mode Decomposition and Hilbert Spectral Analysis, 2008. URL http://cran.r-project.org/web/ packages/EMD/index.html.

D. Nychka. fields: Tools for Spatial Data, 2007. URL http://cran.r-project.org/web/ packages/fields/index.html.
K. Zeng and M-X. He. A Simple Boundary Process Technique for Empirical Mode Decomposition. Proceedings of IEEE International Geoscience and Remote Sensing Symposium, 6:4258-4261, 2004.

Donghoh Kim

Sejong University, Korea

E-mail: donghoh.kimegmail.com

Hee-Seok Oh

Seoul National University, Korea

E-mail: heeseok@stats.snu.ac.kr 